\title{
Microbial Dose-Response Curves and Disinfection Efficacy Models Revisited
}

\author{
Micha Peleg ${ }^{1}$ (D) \\ Received: 25 June 2020 / Accepted: 7 August 2020 / Published online: 28 August 2020 \\ (C) Springer Science+Business Media, LLC, part of Springer Nature 2020
}

\begin{abstract}
The same term "dose-response curve" describes the relationship between the number of ingested microbes or their logarithm, and the probability of acute illness or death (type I), and between a disinfectant's dose and the targeted microbe's survival ratio (type II), akin to survival curves in thermal and non-thermal inactivation kinetics. The most common model of type I curves is the cumulative form of the beta-Poisson distribution which is sometimes indistinguishable from the lognormal or Weibull distribution. The most notable survival kinetics models in static disinfection are of the Chick-Watson-Hom's kind. Their published dynamic versions, however, should be viewed with caution. A microbe population's type II dose-response curve, static and dynamic, can be viewed as expressing an underlying spectrum of individual vulnerabilities (or resistances) to the particular disinfectant. Therefore, such a curve can be described mathematically by the flexible Weibull distribution, whose scale parameter is a function of the disinfectant's intensity, temperature, and other factors. But where the survival ratio's drop is so steep that the static dose-response curve resembles a step function, the Fermi distribution function becomes a suitable substitute. The utility of the CT (or $C t$ ) concept primarily used in water disinfection is challenged on theoretical grounds and its limitations highlighted. It is suggested that stochastic models of microbial inactivation could be used to link the fates of individual viruses or bacteria to their manifestation in the survival curve's shape. Although the emphasis is on viruses and bacteria, most of the discussion is relevant to fungi, protozoa, and perhaps worms too.
\end{abstract}

Keywords Kinetics · Viruses · Bacteria $\cdot \mathrm{CT}$ (or $C t$ ) $\cdot$ Chick-Watson-Hom's models $\cdot$ Distribution functions $\cdot$ Stochastic models $\cdot$ Survival models

\section{Introduction}

COVID 19 [43, 45] transmission through consumption of contaminated food has not been an issue in the current crisis, but other kinds of viruses remain a health hazard [3, 10, 52]. Conventional food preservation methods, primarily targeting cellular organisms especially bacteria, have been apparently efficient in destroying viruses as well [4]. However, meatprocessing plants have recently emerged as hotspots in the pandemic spread in rural areas, and food service operations been identified as potential culprits of its spread among humans. Thus, not surprisingly, disinfection of personnel, produce, clothing, air, tools, equipment, or any surface that humans can be in contact with has recently become a common practice and increasingly mandated by health authorities.

Micha Peleg

micha.peleg@ foodsci.umass.edu

1 Department of Food Science, University of Massachusetts, Amherst, MA 01003, USA
There is a very rich body of scientific and technical literature on disinfection. Being an integral part of medicine, water, and air purification, and an important aspect of the food, pharmaceutical, and other industries, the various aspects of disinfection, including its kinetics, have been extensively investigated at every relevant level form the molecular to the epidemiological.

The objective of this review is neither to evaluate and compare the efficacy of the various available disinfection methods and explain their underlying biological/chemical/ biochemical/physical principles, nor to discuss the engineering aspects of their implementation. This work has the very limited scope to highlight and assess the theoretical implications of the similarities and dissimilarities between kinetic models of water, air and surface disinfection, and those used to quantify the efficacy of food preservation methods, thermal and non-thermal. Although special attention will be given to viruses and bacteria, we should always keep in mind that most of the technologies and agents used for their disinfection are also effective against other biological contaminants. 


\section{Terms and Definitions}

Disinfection, according to the Centers for Disease Control and Prevention (CDC), is a "process that eliminates many or all pathogenic microorganisms, except bacterial spores, on inanimate objects." It differs from sterilization, which "eliminates all forms of microbial life" and from cleaning (also called sanitation) which is "the removal of visible soil (e.g., organic and inorganic material) from objects and surfaces and normally is accomplished manually or mechanically using water with detergents or enzymatic products."

Disinfectants, according to Wikipedia, are "chemical agents designed to inactivate or destroy microorganisms on inert surfaces," which may include fresh produce [26]. But the term also applies to chemical agents used to disinfect water and air [1, 17, 21, 43, 44, 46, 49], ultraviolet (UV) light [23], other forms of radiation [14, 15], and even cold plasma [31].

Dose in our context can be defined as the quantity of disinfectant used in a particular application. Originally, it referred to an amount of medicine prescribed to (dosis in Greek) or of ingested poison by a human. Now, the term is also used for the amount of disinfectant administered to a targeted microorganism or the medium in which it may reside or the surface on which it may be present (see below).

Dose-response curve (or exposure-response relationship) according to Wikipedia "describes the magnitude of the response of an organism, as a function of exposure to a stimulus or stressor after a certain exposure time." The response is commonly expressed as the percent of inactivated organisms and hence has the range of 0 to $100 \%$ (see below).

$L D_{50}$ is the median lethal dose, i.e., the dose that corresponds to a response of $50 \%$ inactivation. It is used to compare disinfectants' potency; the lower the $L D_{50}$ the higher is the agent toxicity or treatment efficacy.

Survival curve in our context is a plot of the number or fraction of surviving organisms (or viable viruses) or its logarithm (almost always 10 based) as a function of the exposure's time, commonly expressed in seconds, minutes or hours. A survival curve obtained under constant lethal agent's intensity and environmental conditions is referred to as static. A survival curve obtained under varying lethal agent's intensity and environmental conditions is referred to as dynamic (see below). Notice that in both static and dynamic exposures to a lethal agent, the survival curve's local slope, which has time reciprocal units, is the momentary ("instant") inactivation rate and hence the connection to kinetics [35].

\section{Issues with the Definition of a Dose}

Ostensibly, the above-listed definitions are all straightforward and their meanings intuitively clear. Yet, this is not always the case with the dose definition, which is a core issue when trying to relate the dose-response curve to the inactivation kinetics. Here is why caution is needed. When dealing with the potency or toxicity of a drug, poison, or any substance ingested by a human or animal, a dose can be quantified in terms and units such as $m g$ ingested $/ \mathrm{kg}$ of the ingesting person or animal. Similarly, the number of a pathogenic microorganism's cells or virus's units ingested by an individual human can also be viewed as a dose. Indeed, a pathogen's virulence and infectivity, which determine the severity of the damage that it causes and the speed of its spread, are intimately associated with the number of ingested cells or virus units needed for acute infection. In contrast, in water or air disinfection, the disinfectant's effective concentration is determined in the medium where the targeted pathogen resides and not in the microorganism or virus itself. Similarly, in surface disinfection by an active chemical compound or radiation, the treatment's intensity is expressed in terms of mass or energy per unit treated area and not per the targeted individual microbe's cell or virus unit, or a specified number of the targeted cells or units, or even their biomass, which would depend on the contamination level and its pattern.

Moreover, rarely is the desired disinfection level goal, expressed as the number of decades reduction in the targeted microbial population's size, is accomplished instantaneously. Consequently, the exposure's duration becomes a crucial consideration and ought to be taken into account in the treatment's intensity quantification. This is manifested in the CT, or $C t$, concept [32] according to which a chemical disinfectant' effective dose is expressed as the multiplication product of the disinfectant's concentration $(C)$ and the exposure time ( $T$ or $t)$ needed to reduce the targeted microbial population's by a chosen number of decades, an issue to which we will return. (The confusing traditional CT term, where $T$ represents time and not temperature (and which has nothing to do with the State of Connecticut...), is a carry on from an older publication where the integration limit of $t$ was assigned the letter $T$.)

Also, in chemical disinfection processes, the administered lethal agent is a highly active chemical compound, e.g., ozone, chlorine dioxide, or paracetic acid. Such compounds are chemically unstable; the primary cause of their reactivity and rationale of their use as disinfectants. But as a result, their effective concentration diminishes with time, sometimes requiring their constant or periodical replenishment. The gaseous disinfectants are also volatile and hence, their effective concentration can diminish even without chemically disintegrating or reacting with the targeted microbe. Either way, maintaining a predetermined constant concentration of such chemical agents during the disinfection process, or even in studies of its kinetics in the laboratory, is technically difficult. This makes the experimental determination of perfectly static survival curves, and similarly dose-response curves, a challenging task. It also raises the question of whether the initial or average concentration is or can be an acceptable representative of the diminishing effective concentration. 
The above issues, especially how to account for the time's role in disinfection mathematically, have been and can be tackled in various ways, which we'll address in what follows. Suffice it to state at this point is that since time is inherently involved in both dose-response and survival curves, each cannot be considered in isolation but as a manifestation of the same underlying inactivation kinetics.

\section{Viral Versus Bacterial Inactivation}

There is an ongoing debate on whether a virus can be considered a life form but it should not concern us here. From a mathematical modeling viewpoint, the inactivation of a viral, bacterial, or other microbial population follows similar kinetics despite that the underlying mechanisms at the individual cell/unit and molecular levels can be quite different. Since in what follows, we'll only address the kinetics at the population level, the focus will be on concepts and models that are pertinent to the disinfection of either or both viruses and bacteria.

There are two main differences between viral and other organismic populations that should be always kept in mind:

1. Unlike at least certain kinds of bacteria, viruses do not have damage repair mechanisms. Therefore, unless proven otherwise, their inactivation can be viewed as irreversible. It will be assumed, however, as has presumably been done in the cited publications, that the same applies to bacteria and other pathogens on the pertinent time scale. In other words, we will assume that issues concerning injury, repair, and/or adaptation need not be taken into account for modeling the disinfection kinetics.

2. Unlike in a decimated bacterial and other microbial population, the number of survivors in a viral population, if any, cannot rise without recontamination. In other words, depending on conditions, the survivors of a bacterial or other microbial population can resume cell division. Thus, at least in principle, their numbers can not only rise but also exceed the original contamination level. This demonstrated in Fig. 1. It shows the screen display of a freely downloadable Wolfram Demonstration that can be used to simulate such and other inactivation/growth scenarios (open: https://demonstrations.wolfram.com/ GrowthInhibitionAndRetardationByAntimicrobials/). [To run the Demonstration and download, the also freely downloadable Wolfram CDF Player, which runs it and more than 12,000 other Demonstrations to date, follow instructions on the screen.] We will also assume that for the time scale pertinent to disinfection the issue of spore germination need not be addressed.

There can also be differences between how long viruses, bacteria, and other microorganisms remain viable when dispersed in untreated air or water, or on a contaminated surface. These differences can vary dramatically depending on the virus or organism type, the particular habitat, and on the ambient conditions, notably temperature, and in air or on a surface on the relative humidity too. It has been traditionally assumed though that the rate of a pathogen perishing spontaneously is very small relative to that induced by a disinfectant, and therefore need not be taken into account in dose-response or survival curves modeling. Nevertheless, although true, we will still show how natural attrition can be incorporated into a survival model if needed.

\section{Dose-Response Models}

The qualitative difference between a dose of ingested substance or pathogen by a human, or any organism for this matter, and a dose administered externally to a targeted microbe or virus as in disinfection, has been explained in previous sections. Let us call the dose-response curve based on the first kind "type I," and based on the second "type II." It so happened that the type I dose-response curves are traditionally described mathematically and represented graphically as a relationship between the rising fraction (or \%) of the infected or dead individuals as a function of the ingested dose, or its logarithm, as shown schematically in Fig. 2 (left). In contrast, type II dose-response curves are commonly described mathematically and presented graphically as a relationship between the falling number (or fraction) of the treatment's survivors, or its logarithm, and the dose administered to the medium in which they are imbedded or the surface on which they reside as shown schematically in Fig. 2 (right). The drop in the number of survivors can be gradual (solid curve) or very steep, almost instantaneous (dashed curve), as can be seen in the figure.

\section{Mathematical Models of Type I Dose-Response Curves}

The ubiquitous sigmoid shape of type I dose-response curves has been described mathematically by a variety of algebraic models. The most notable among them are the two parameters beta-Poisson function [1, 22, 25, 27, 30, 48, 55], an example of which is given in Fig. 2 (left). In its simplified version [55], this model has the form:

$P($ Dose $)=1-\left[1+\frac{\text { Dose }}{\beta}\right]^{-\alpha}$

where the Dose refers to the ingested drug, poison, etc., usually expressed on per kilogram body weight or similar weight basis, and $\alpha$ and $\beta$ are constants. The magnitudes of $\alpha$ and $\beta$ 
Fig. 1 Screen display of a Wolfram Demonstration that generates survival curves turned into growth curves of the survivors

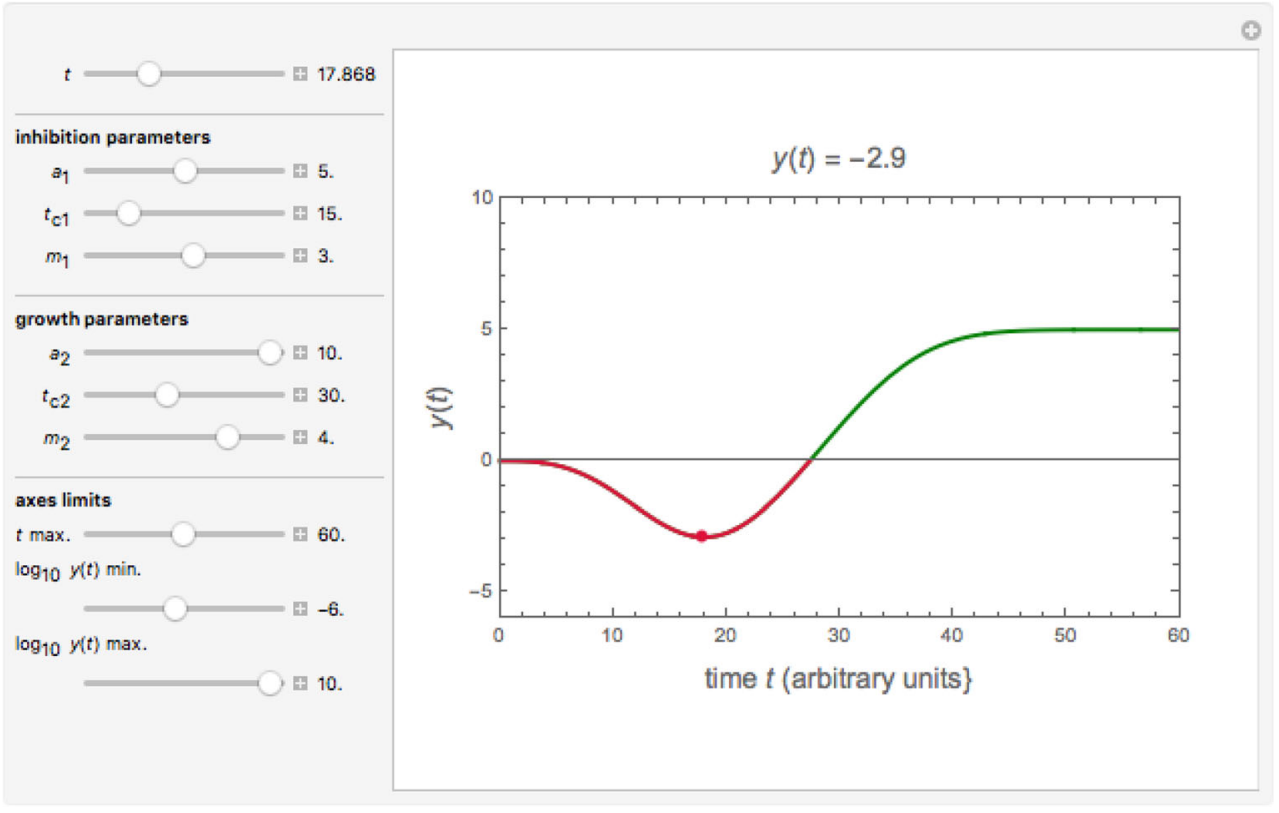

depend on the toxic material kind and the manner in which it is ingested. According to this model $L D_{50}=\beta /\left(2^{1 / \alpha}-1\right)$.

The lethal or infectious Dose can also be the number $N$ of ingested, inhaled, or injected virus units or a pathogenic bacterium's cells in which case the model will assume the form:

$P(N)=1-\left[1+\frac{N}{\beta_{N}}\right]^{-\alpha_{N}}$

where $P(N)$ is the probability (expressed as a fraction or \%) of death or acute infection, and $\alpha_{N}$ and $\beta_{\mathrm{N}}$ are constants whose magnitude depends on the kind of pathogen and the manner in which it enters the body. The beta-Poisson is not a unique model. It can be shown that data generated with it can be fitted with the cumulative form of the lognormal distribution (open and use the interactive Wolfram Demonstration https:// demonstrations.wolfram.com/PathogenDoseResponse CurvesWithTheBetaPoissonAndLognormalMode/), as demonstrated in Fig. 3, and most probably with other unimodal distribution functions.
The interchangeability of the beta-Poisson and lognormal distribution functions for dose-response data representation is not a coincidence. It can be shown mathematically and through Monte Carlo simulations that if the mortality probability $\left(P_{N}\right)$ is the multiplication product of a set of underlying probabilities of several events $\left(P_{i} \mathrm{~s}\right)$, i.e., $P(N)=\prod P(i)$, and if each of these underlying $P(i)$ is uniformly distributed within its specific range, then the resulting probability of infection $\left(P_{\mathrm{N}}\right)$ has a lognormal distribution, which is indistinguishable from the beta-Poisson for all practical purposes (see https:// $\mathrm{d}$ e mon s t rat i o n s. wo $1 \mathrm{fram}$. c o m/ ExpandedFermiSolutionsInPathogensDoseResponseCurves/ and $[38,41,42])$. The Wolfram Demonstrations' screen display is shown in Fig. 4. More elaborate software to analyze dose-response relationships is described in [55].

For microbial and viral infections, an alternative to the beta-Poisson model is the single-parameter exponential model:

$$
P(N)=1-\operatorname{Exp}\left[-k_{N} N\right]
$$

Fig. 2 Type I and type II doseresponse curves
Type I

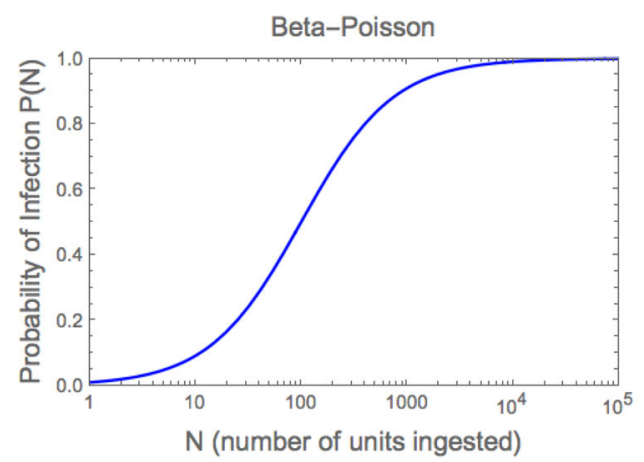

Type II

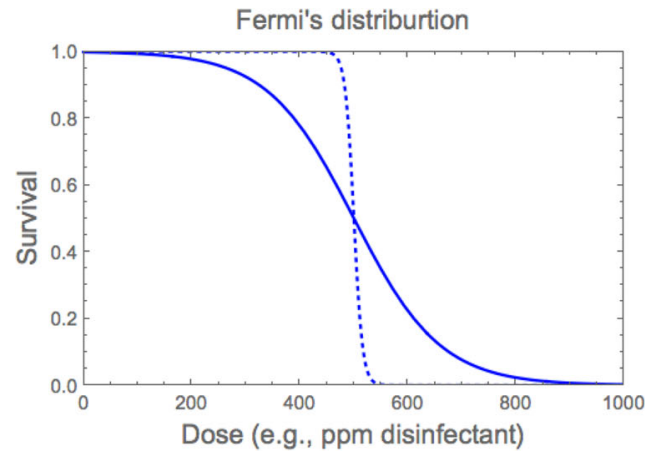


Fig. 3 Screen display of a Wolfram Demonstration that shows the interchangeability of the beta-Poisson and lognormal distributions

Fig. 4 Screen display of a Wolfram Demonstration that shows how a type II doseresponse curve emerges when the probability of acute infection is the multiplication product of underlying factors probabilities each having an assumed range. Generated with the Monte Carlo method

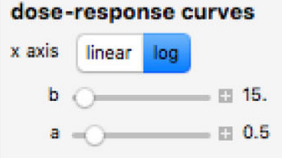

seed repeatable random numbers $\checkmark$

seed value

C 1

noise amplitude ( $\%$ of full scale) - It 5.

number of points to generate $-0=20$

ingested number axis maximum $-\mathrm{O}=\mathrm{O} 500000$. fitted parameters: $\quad \mu_{L}=4.035 \quad \sigma_{L}=2.314 \quad r^{2}=0.999$

scattered dose-response data fitted with lognormal modı

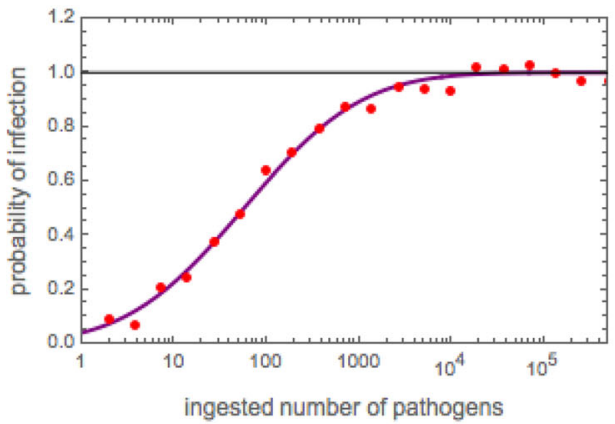

beta Poisson function used to generate the data

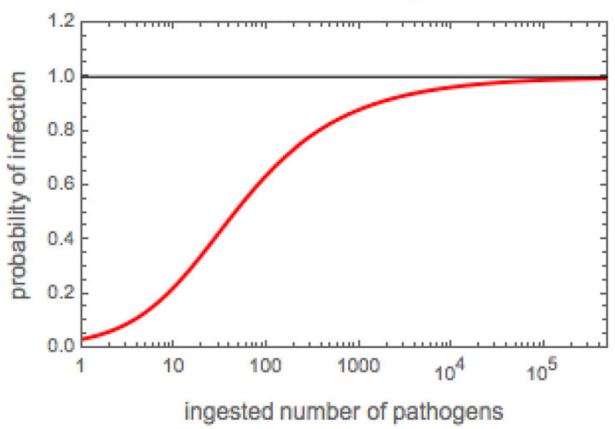

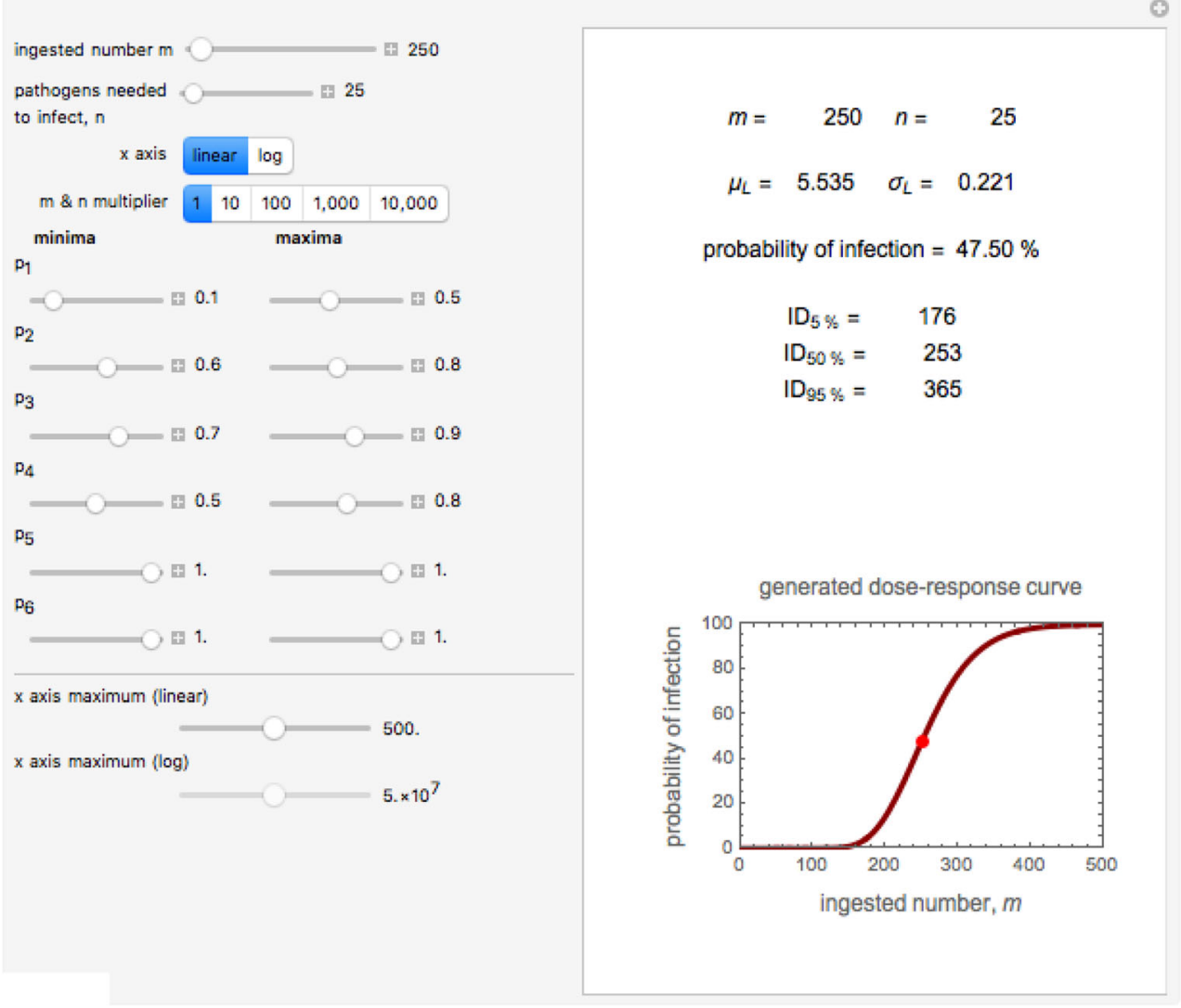


where $k_{\mathrm{N}}$ is a characteristic constant whose magnitude depends on the pathogen and the way it enters the body. A hypothetical dose-response curve generated with this model is shown in Fig. 5 (left). Equation 3 too is not a unique doseresponse model, and its fit to experimental data could most probably be matched by a Monod type model

$P(N)=\frac{N}{b_{N}+N}$

where $b_{\mathrm{N}}$ is the adjustable parameter. (See the Wolfram Demonstration https://demonstrations.wolfram.com/ CharacteristicTimesInAccumulationAndDecay/). An example of a dose-response curve, generated with Eq. 4 as a model, is given in Fig. 5 (right). If and where found applicable, the advantage of this non-exponential model over the beta-Poisson and exponential models would be that $b_{\mathrm{N}}=$ $L D_{50}$. (This is because at $P(N)=1 / 2, N=b_{\mathrm{N}}$.)

For technical and logistic reasons, experimental viral and bacterial dose-response data of type I are usually few in number and notoriously widely scattered, frequently showing obvious outliers (e.g., $[22,53,56])$. Therefore, any preference of one model over another (even having the same number of adjustable parameters) based on statistical fit alone should be viewed with caution. Also, notice that the sigmoid shape of the dose-response curves described by Eqs. 1-4 as models only appear when drawn as linear-log plots as shown in Fig. 2 (left) and Fig. 5. Thus, to generate a sigmoid dose-response curve on linear coordinates would require different kinds of models. One possible candidate for such a case would be the two-parameter version of the exponential model known as the stretched exponential model, which would have the form

$P($ Dose $)=1-\operatorname{Exp}\left[-k_{\text {Dose }}\right.$ Dose $\left.e^{n_{\text {Dose }}}\right]$

or

$P(N)=1-\operatorname{Exp}\left[-k_{N} N^{n_{N}}\right]$

where the subscripts Dose or $N$ denote that the ingested dose is of a chemical compound or a number of pathogen cells or units, respectively. The stretched exponential model is akin to the cumulative form of the Weibull distribution, which can sometimes be used interchangeably with the Fermi distribution. Both will be discussed in more detail in the next sections.

Since the focus of this review is on the mathematical properties of dose-response curves and disinfection kinetics, models of microbes' pathogenicity when orally ingested, inhaled, or entering the body by other means (e.g., through insect bites or injection) will not be discussed. However, we will return to statistical distributions as determining the shape of type II dose-response curves and that of microbial survival curves, which are used to assess the efficacy of food preservation methods.

\section{Type II Dose-Response Curves}

Intuitively, one would expect that because a type II doseresponse curve depicts the survival ratio (instead of the destroyed fraction) versus dose relationship, it would be simply a flipped or mirror image of that of type I, replacing the infected humans or animals with the targeted microbes. This is not always the case, however, for two major reasons: The applied disinfectant's intensity (concentration, radiation energy, etc.) is intentionally set at a lethal level, and time plays a more central role. In fact, type I dose-response curves are sometimes determined at different days after the infection or inoculation (see [56]), while disinfection usually takes place on a much shorter time scale. As can be seen in Fig. 2 (right), the survival ratio's drop with the dose increase, however the dose is defined, could vary between gradual (solid line) to almost perfectly vertical, and with (dotted line) or without a noticeable "shoulder." Either way, the similarity of the type II dose-response curves' shapes to those of the more familiar microbial survival curves encountered in thermal and nonthermal inactivation is unmistakable. This should not come as a surprise because both reflect a common characteristic response of biological populations to a hostile environment. Had all the targeted microbes been genetically identical and in exactly the same physiological state, i.e., having the same vulnerability, and had the lethal agent been applied in perfect uniformity, then, at least in principle, they would all succumb
Fig. 5 Simulated type I doseresponse curves generated with two empirical models (Eqs. 3 and 4), alternatives to the beta-Poisson distribution
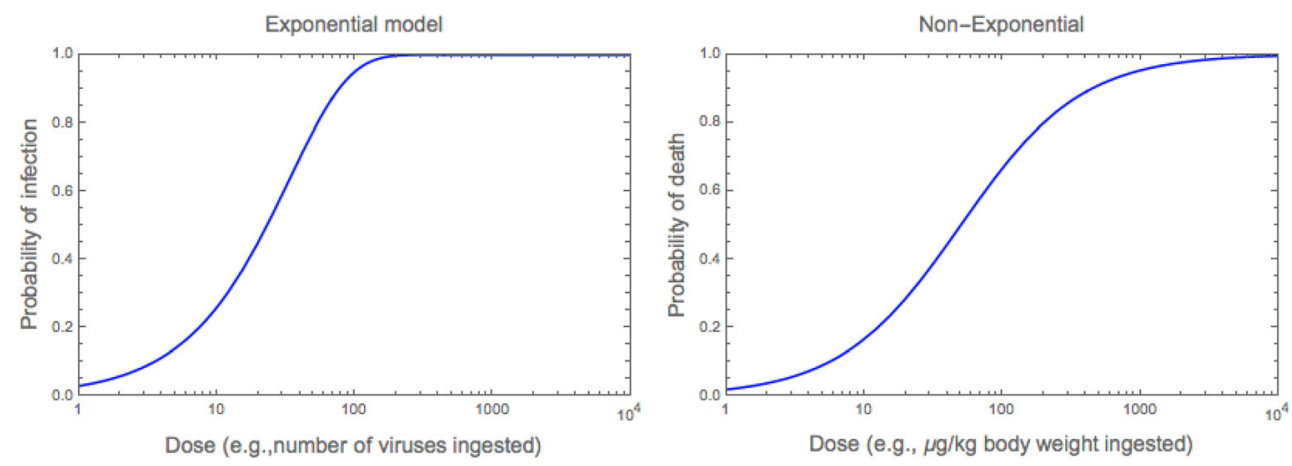
to the very same dose at exactly the same time. The doseresponse curve in such a case would be a perfect step function [33-35] very similar to that shown as a dotted curve in Fig. 2 (right). The corresponding dose magnitude in such a case would be a measure of the individual virus unit or bacterial cell's sensitivity to or tolerance of the lethal agent, which all members of the population share and exhibit in unison, or vice versa. A dose-response curve having the shape of a perfect step function would indicate that the above-mentioned conditions are fully satisfied; i.e., that all the targeted viruses or bacterial cells are indeed identical and they succumb simultaneously to the uniformly applied disinfectant. Obviously, this is rarely the case in practice and most type II dose-response curves indicate that there is an underlying narrow or wide spectrum of sensitivities and/or at least a certain degree of non-uniformity in the exposure's intensity [39]. Let us emphasize that for a true step dose-response curve to appear, both uniformity conditions ought to be satisfied simultaneously. But viruses can at least sometimes exhibit slight compositional variations $[47,48]$ and their dispersion pattern might not be uniform due to aggregation, for example [5, 29]. Residing on a non-uniform surface may also cause non-uniform exposure. Bacteria and other microorganisms, even if their dispersion is uniform can be in different physiological states as a result of age, cell division stage, etc. Consequently, type II doseresponse curves closely resembling a step function are uncommon [33-35]. The concept of an underlying spectrum of sensitivities or resistances also applies to the various shapes of survival curves (see below) except that their primary manifestation is in the survival ratio's dependence on time.

\section{Prominent Flat Shoulder}

A prominent flat shoulder in a type II dose-response curves (of the kind shown in Fig. 2 (right)) has been reported in various publications (e.g., $[8,32])$. The shoulder's presence has been commonly considered a special case where the drop in the survival ratio commences only after the administration of a critical dose or as a consequence of a characteristic physiological lag time. In other words, the idea has been that there are two distinct response regimes and a sharp transition between them. An alternative view is that both type II dose-response curves (of the kind shown in Fig. 2 (right)) and survival curves encountered in thermal food preservation having a similar shape are actually the cumulative form of an underlying unimodal distribution of the targeted microbe's sensitivities to the imposed lethal agent. The sensitivities (or resistances) are manifested in the dose needed to inactivate the microbe in the former, or the time it takes to inactivate it in the latter. From this viewpoint, the primary difference between a doseresponse and a survival curve is that in the first, the survival ratio is presented as a function of dose while in the second as a function of time. If this observation is correct, then a prominent flat shoulder will always appear if the mode of the sensitivities distribution is considerably larger than its variance $[34,35]$. In other words, as long as the underlying distribution is unimodal, the distribution kind is immaterial; it can be normal, lognormal, Weibull, log-logistic, Fermi, or whatever. This is demonstrated in Fig. 6. The figure shows two pairs of juxtaposed curves generated with the Weibull and Fermi distributions, left and right respectively. In each pair, the distribution's spread (variance) is about the same but only when it is considerably smaller than the mode does a prominent shoulder appear. Despite their superficial similarity, the initially very mild drop in a concave downward semilogarithmic Weibullian dose-response or survival curve (having a shape factor larger but close to one; see below) should not be confused with a shoulder. Thus, any suggestion that it represents a special physiological state or inactivation mechanism ought to be backed up by independent experimental evidence.

\section{Prominent Flat Shoulder with Log-Linear Continuation}

A special case of a flat shoulder in a dose-response or survival curve is where the continuation is clearly log-linear as shown in Fig. 6 (right) [35, 37]. This pattern has been traditionally interpreted as a delayed first-order decay kinetics triggered when a threshold dose or characteristic lag time is crossed. Here, again (see figure), the alternative explanation is that there is no abrupt transition between two regimes. According to this second view, the dose-response or survival curve's shape is determined by an underlying spectrum of sensitivities that follows the Fermi distribution $[33,35]$. If true, then because the smoothly changing slope takes place in a relatively small region, it is likely to be missed when the dose or survival curve is constructed from spaced experimental data. More details on the mathematical properties of the Fermi and Weibull distribution functions will be provided in a later section.

\section{Traditional Kinetic Survival Models for Microbial Disinfection}

\section{The Chick-Watson Model}

The old Chick-Watson model $[7,9,54]$ is probably the most well known and widely used to describe the kinetics of disinfection by a chemical agent [19-22]. For static, i.e., constant disinfectant concentration, it can be written in the form

$\log [S(t)]=\log \left[\frac{N(t)}{N_{0}}\right]=-k C^{n} t$ 
Fig. 6 "Long flat shoulder" in the cumulative form of unimodal distributions when their mode is much larger than their variance
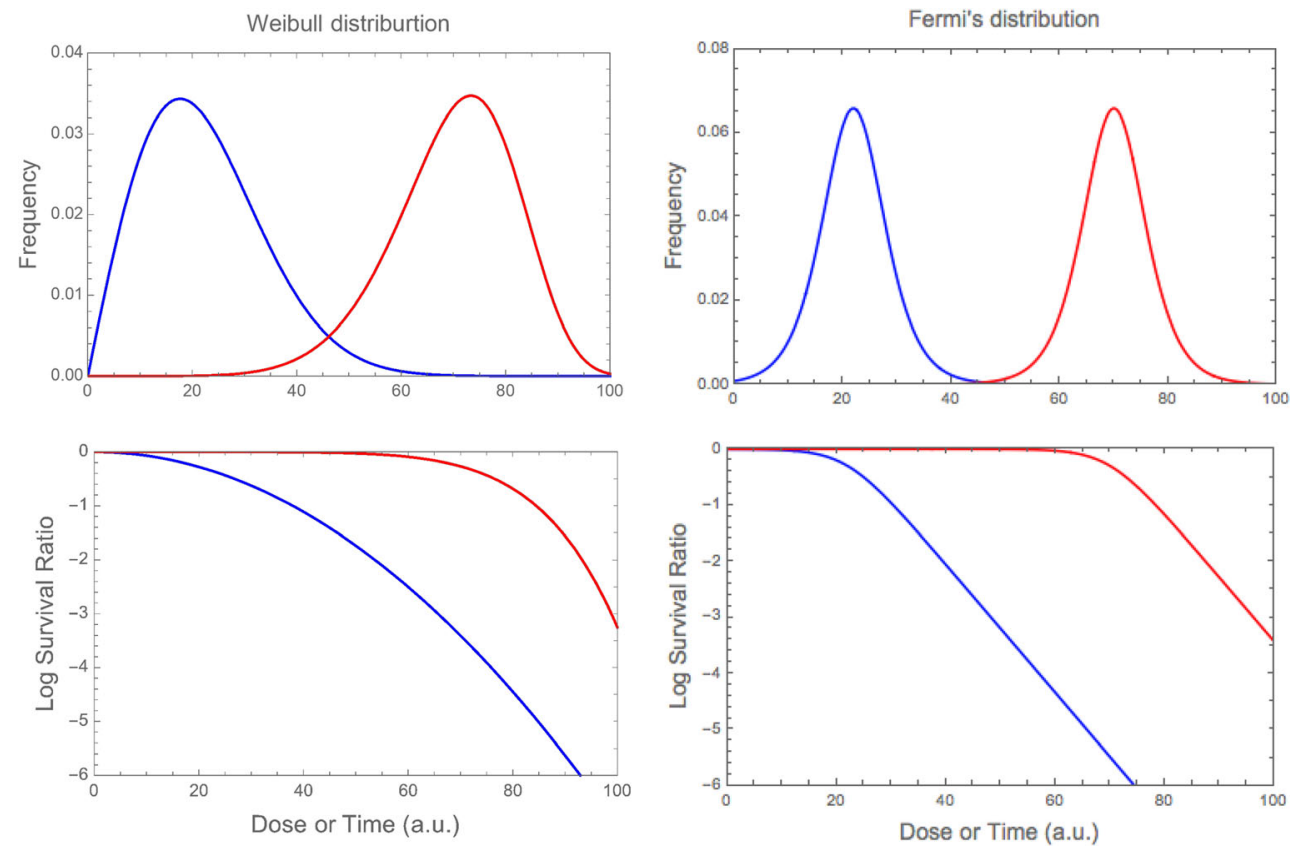

where $S(t)$ is the survival ratio, defined as $N(t)$, the number of viable survivors after time $t$, divided by $N_{0}$, the initial number of the targeted virus units or microbial cells, and $C$ is the "residual disinfectant concentration." The model's two adjustable parameters, $k$ and $n$, are characteristic to the targeted microbe and the particular disinfectant. Their magnitudes depend on the type of medium where the disinfection takes place and ambient conditions, notably temperature and $\mathrm{pH}$, or relative humidity where relevant. Whether $\log [S(t)]$ 's base is $e$ or 10 is immaterial and will be only manifested in the numerical value of the corresponding $k$.

The rate version of the Chick-Watson model according to the literature [19, 32] has the form

$\frac{d N(t)}{d t}=-k C^{n} t$

From a formal viewpoint, Eq. 8 describes a static first-order decay or loss kinetics where the characteristic rate constant $\boldsymbol{k}$, in time reciprocal units, is proportional to the momentary disinfectant's concentration raised to the power $n$, i.e., $\boldsymbol{k}=k C^{n}$. To avoid confusion, let it be understood that Eq. 8 in the above form only applies to a constant disinfectant concentration, or in other words, it can only describe the targeted microbe's decay rate if and only if the disinfectant concentration remains unchanged during the disinfection (see below).

\section{Hom's Model}

An expanded version of the Chick-Watson survival model is known as Hom's model, which for static disinfection can be written in the form [21]. $\log [S(t)]=\log \left[\frac{N(t)}{N_{0}}\right]=-k C^{n} t^{m}$

where the three parameters are $k$ and $n$ transplanted from the Chick-Watson model, and $m$ an added exponent. The original Chick-Watson equation is therefore a special case of the Hom's model where $m=1$.

According to various publications (e.g., [18-20]), Hom's model underlying differential rate equation is

$\frac{d N(t)}{d t}=-k m C^{n} t^{m-1} N(t)$

Although apparently intended to describe dynamic disinfection, the above form of Hom's model, like its predecessor Eq. 8, is only applicable to processes where the disinfectant concentration remains constant throughout the entire disinfection process. In other words, Eq. 10 can describe the targeted microbe's elimination rate if and only if the disinfectant's concentration and hence the term $C^{n}$ is not a function of time. If, however, the residual disinfectant concentration falls, rises (through replenishment) and/or oscillates, that is varied and becomes a function of time, i.e., $C=C(t) \neq$ constant, then the governing differential rate equation ought to be

$\frac{d \log [S(t)]}{d t}=-k t^{m-1} C(t)^{n-1}\left[m C(t)+n t \frac{d C(t)}{d t}\right]$

where the actual time $t$ is the same as the time $t^{*}$ (see below), which corresponds to the momentary survival ratio, i.e., the (numerical) solution for $t$ of the equation

$\log [S(t)]=-k^{*} C(t)^{n} t^{m}$ 
(For details see [13, 35] and below.)

Despite its cumbersome appearance, Eq. 11, especially with $t^{*}$ being a momentary numerical solution of an algebraic equation, is still an ordinary differential equation (ODE). Thus, although yet be attempted, one can expect that with modern mathematical software Eq. 11 would be solved numerically for almost any conceivable concentration profile $C(t)$ expressed algebraically. At least in principle, if and when the Chick-Watson or Hom's model is validated experimentally, Eq. 11 could be used to simulate or predict the outcome of realistic actual or contemplated dynamic disinfection processes, where the disinfectant concentration does vary with time.

The reported applicability of the Chic-Watson-Hom's models, in their original and modified versions, seems to have been primarily based on their respective equations' fit to experimental static data, rather than to their ability to predict dynamic survival patterns. Unfortunately, good fit by statistical criteria alone cannot be considered a model validation. The fit only provides evidence that the proposed model can describe the observed data at hand mathematically and does not even establish its uniqueness. Proper validation of a survival model, or of any kinetic model for that matter, requires that it can predict correctly survival data not used in its parameters determination, for example, predicting correctly dynamic survival curves from static data or vice versa, or dynamic survival data from other dynamic survival data $[13,35$, 37].

\section{Models Based on an Underlying Spectrum of Sensitivities}

Alternatives to the Chick-Watson-Holm's type survival models are the already mentioned models based on an underlying spectrum of sensitivities or resistances described by a distribution function. Several such distribution functions can be considered. The first two natural candidates that come to mind are the commonplace and highly flexible Weibull and lognormal distributions, or for the less common case of an extremely narrow distribution, where the dose-response or survival curve resembles a step function, the less familiar Fermi distribution becomes a promising option [33], as demonstrated in Fig. 2.

\section{The Weibull Distribution}

The Weibull distribution has been used in the mathematical description of a wide range of failure phenomena and hence its ubiquitous association with risk assessment in a variety of technological fields. Since the inactivation or death of an individual microbe during disinfection can be viewed as its failure to overcome the destructive or lethal agent, the Weibull distribution is a natural choice $[13,35]$. The two parameters of the more familiar form of the distribution function named after Weibull are $\alpha$ known as the shape factor and $\beta$ known as the scale factor. For our purpose, since we deal with a process's kinetics, we will use the model's version known as the RosinRammler distribution, which had been proposed (to particlesize reduction operations) 3 years prior to its introduction by Weibull [6].

For static disinfection (constant disinfectant concentration), it can be written in the form $[2,13,35]$.

$\log [S(t)]=-b(C) t^{m(C)}$

where $b(C)$ is a concentration-dependent rate parameter, related to Weibull's scale factor reciprocal, and $m(C)$ a power that can but need not be concentration-dependent, equivalent to Weibull's shape factor. Where and when $m(C)$ is independent or practically independent of the concentration, which is not uncommon (see $[49,50,51]$ ), the static model becomes

$\log [S(t)]=-b(C) t^{m}$

This equation is reminiscent of Hom's model, except that the rate parameter $b(C)$ need not be in the form of the powerlaw expression. In other words, Hom's model can be viewed as a special case of the Weibullian model where $b(C)=k C^{n}$.

Implementing the assumption that in dynamic disinfection, where $C=C(t)$, not a constant, the momentary inactivation rate is the static rate at the disinfectant's momentary concentration, at the time that corresponds to the momentary survival ratio [13, 35], renders the rate model

$\frac{d \log [S(t)]}{d t}=-b[C(t)] m\left[\frac{-\log [S(t)]}{b[C(t)]}\right]^{(m-1) / m}$

Here, again, despite its cumbersome appearance, Eq. 15 is still an ordinary differential equation (ODE). Therefore, as has been already demonstrated [ibid], it can be rapidly solved numerically with modern mathematical software for almost any conceivable relevant concentration history $C(t)$ expressed algebraically, including containing If statements to account for instant replenishments. Thus, at least in principle, this model can replace the Chick-Watson-Hom type models when validated experimentally by testing its predictive ability. Examples of Eq. 15's solutions for disinfection with a dissipating agent can be generated with a freely downloadable interactive Wolfram Demonstration https://demonstrations. w o $1 \mathrm{f} \mathrm{r} \mathrm{a} \mathrm{m.} \mathrm{c} \mathrm{o} \mathrm{m/}$

MicrobialSurvivalWithDissipatingDisinfectant/. Its screen display is shown in Fig. 7.

According to the Weibullian model [35], if the shape factor, i.e., the power $m$, is larger than one $(m>1)$, the static survival curve has downward concavity, which suggests that 
Fig. 7 Screen display of a Wolfram Demonstration that simulates microbial survival curves during exposure to a chemically unstable or volatile disinfectant

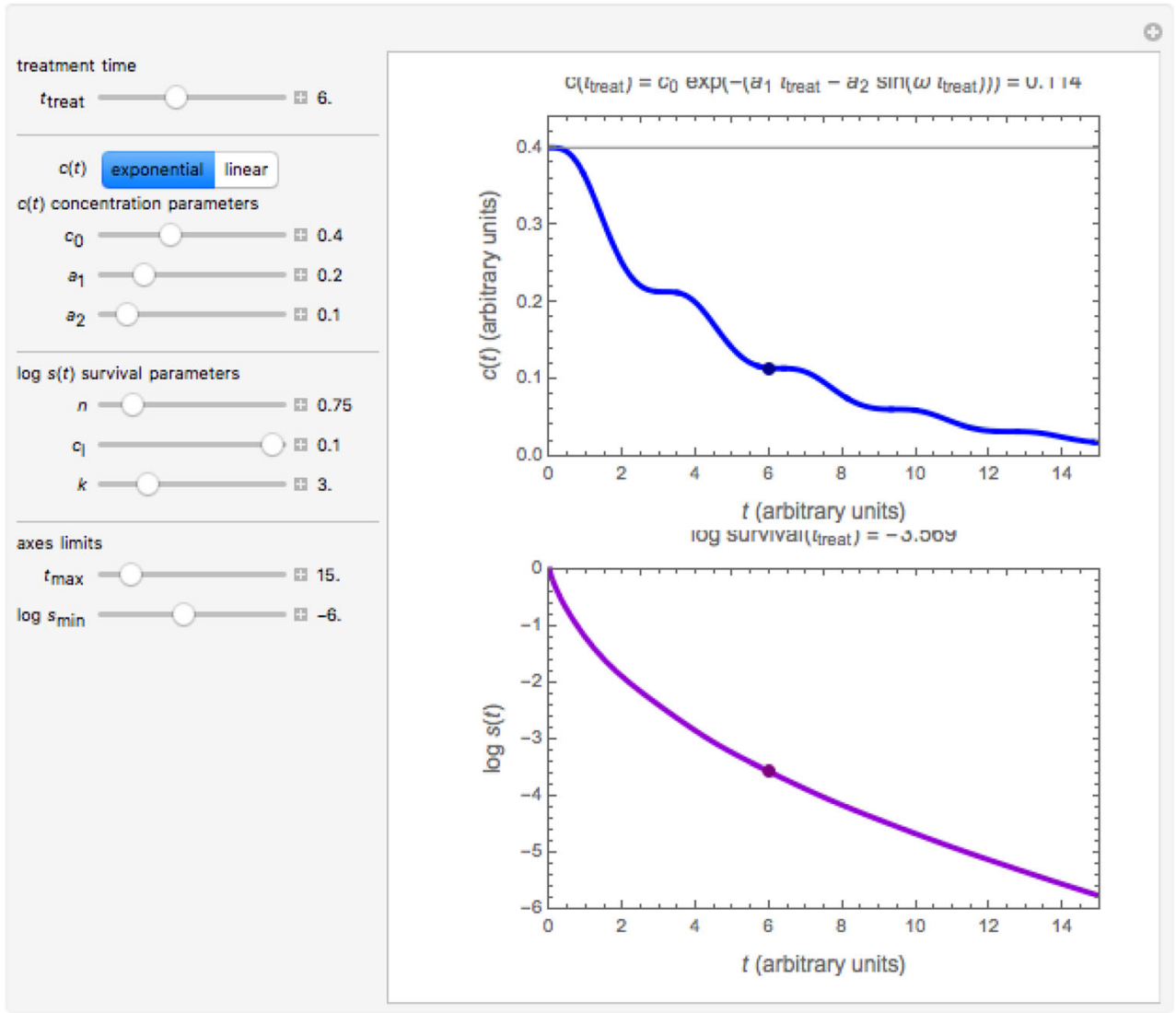

damage accumulation progressively weakens the survivors' resistance to the lethal agent. Where $m<1$, the survival curve has upward concavity, a shape known as tailing [28, 46]. Its observation suggests that sensitive members of the targeted population are quickly eliminated leaving progressively sturdier, i.e., more resistant survivors. What's known as first-order or log-linear kinetics is a special case of the Weibullian model where $m=1$. Notice that the local slope of the cumulative form of the Weibullian distribution, or that of any alternative distribution function for this matter, is the momentary survival or inactivation rate having time reciprocal units. In other words, the notions of a spectrum of resistances or sensitivities characterized by a distribution function and of inactivation kinetics are the two sides of the same coin.

\section{The Expanded Weibullian Model}

Assuming that the shape factor $m$ remains constant or practically constant, the rate parameter $b[C]$ in Eq. 14 or $b[C(t)]$ in Eq. 15 is solely a function of the disinfectant's residual concentration. However, and regardless of how it is expressed, the magnitude of its parameters also depends on other factors, most notably the temperature, $T$, which too can vary considerably during a long disinfection process. The rate constant's dependence on simultaneously varying disinfectant concentration and the temperature is rarely if ever known a priori.
Also, there is no reason to assume that their combined effect is somehow multiplicative (the basis of the "gamma hypothesis"). Traditionally, the temperature effect has been assumed to follow the Arrhenius equation despite that its universal applicability cannot be taken for granted for several reasons $[16,39]$. The same is true for alternative temperature dependence models. In an assumption-free model where only the concentration and temperature play major roles, the static survival rate parameter can be written as $b(C, T)$. If so, then in dynamic disinfection the momentary disinfection can be written in a nested form [35], i.e., $b(t)=b[C[T(t)]]$ or $b(t)=$ $b[T[C(t)]]$. In the nested forms, the concentration effect on the rate parameter can be temperature-dependent, or alternatively, the temperature effect on the rate parameter can be concentration-dependent. To illustrate this notion, consider the oversimplified example of a hypothetical (unlikely but not totally unrealistic) scenario where a virus's Weibullian rate parameter rises linearly with the disinfectant concentration. If so, we can write $b(C)=k_{0}+k_{\mathrm{C}} C$, where $k_{0}$ represents its natural disappearance rate without the disinfectant presence (if not totally negligible) and $k_{\mathrm{C}}$ the $b(C)$ versus $C$ relationship's slope. For simplicity, let us assume that $k_{0}$ is very small and practically unchanged and that in the pertinent temperature range $k_{\mathrm{C}}$ rises with temperature following the exponential model $k_{\mathrm{C}}=k_{\mathrm{CTref}} \operatorname{Exp}\left[k_{\mathrm{T}}\left(T-T_{\text {ref }}\right)\right]$, where $k_{\mathrm{CTref}}$ is the rate constant at a chosen reference temperature within the pertinent 
range. Both $T$ and $T_{\text {ref }}$ are in degree Celsius. [Curves generated with this simple model are practically indistinguishable from those produced by the Arrhenius equation [39], and their construction needs no conversion of the temperatures to degree Kelvin terms.] Where both the disinfectant's concentration and temperature vary simultaneously, the momentary Weibullian rate constant, $b(t)$, becomes $b(t)=k_{0}+$ $k_{\mathrm{CTref}} \operatorname{Exp}\left[k_{\mathrm{T}}\left(T(t)-T_{\text {ref }}\right)\right] C(t)$. Thus, with the assumption that $k_{0}$ and $m$ are practically independent of both concentration and temperature, the dynamic survival model becomes

$\frac{d \log [S(t)]}{d t}=-b(t) m\left[\frac{-\log [S(t)]}{b(t)}\right]^{(m-1) / m}$

where $b(t)$ is the expression in which both the concentration profile, $C(t)$, and temperature profile, $T(t)$, are incorporated.

Dynamic survival curves generated with Eq. 16 as a model are given in Fig. 8.

A similar rate equation can be constructed for more realistic and more complicated temperature or concentration dependencies, which may include terms of $k_{0}(t)=k_{0}[C[T(t)]]$ and $m(t)=m[C[T(t)]]$. The same can be said about $b(t)=$ $b[T[C(t)]]$ and $k_{0}[T[C(t)]]$ and $m(t)=m[T[C(t)]]$, whichever might be the more convenient.

The issue here is not the complexity of the resulting rate model's equation. For almost all conceivable $C(t)$ and $T(t)$ histories, Eq. 16 will remain ODE which could be solved numerically almost instantaneously. The issues are primarily logistic and of two kinds: Unlike temperature recording, monitoring the disinfectant's residual concentration at close enough time intervals may not be a simple matter, and to determine experimentally the model's parameters, $k_{0}, k_{\mathrm{CTref}}$, and $k_{\mathrm{T}}$, even in the simplified example above may not be a feasible option. The problem will be further aggravated if a more realistic and hence more elaborate $b[C[T(t)]]$ or $b[T[C(t)]]$ expression is employed. The resulting model's terms will require the experimental determination of even more parameters, rendering the whole endeavor impractical. The situation will become almost totally unmanageable if terms accounting for the roles of additional factors such as $\mathrm{RH}$ or $\mathrm{pH}$ are also incorporated in the model's rate equation.

In light of the above, it is highly doubtful that the method as described will be implemented in disinfection studies any time soon. But the underlying concept should not be thrown overboard for two reasons: Models with assumed parameters can be used in simulations to reveal patterns (especially as a result of oscillations) that might not be obvious, and keeping the conceptual model in mind will help to avoid making assumptions about the kinetics that are difficult to defend.

The simulated oscillating concentration and temperature profiles and corresponding survival curves shown in Fig. 8 demonstrate these points. The three plots at the bottom show
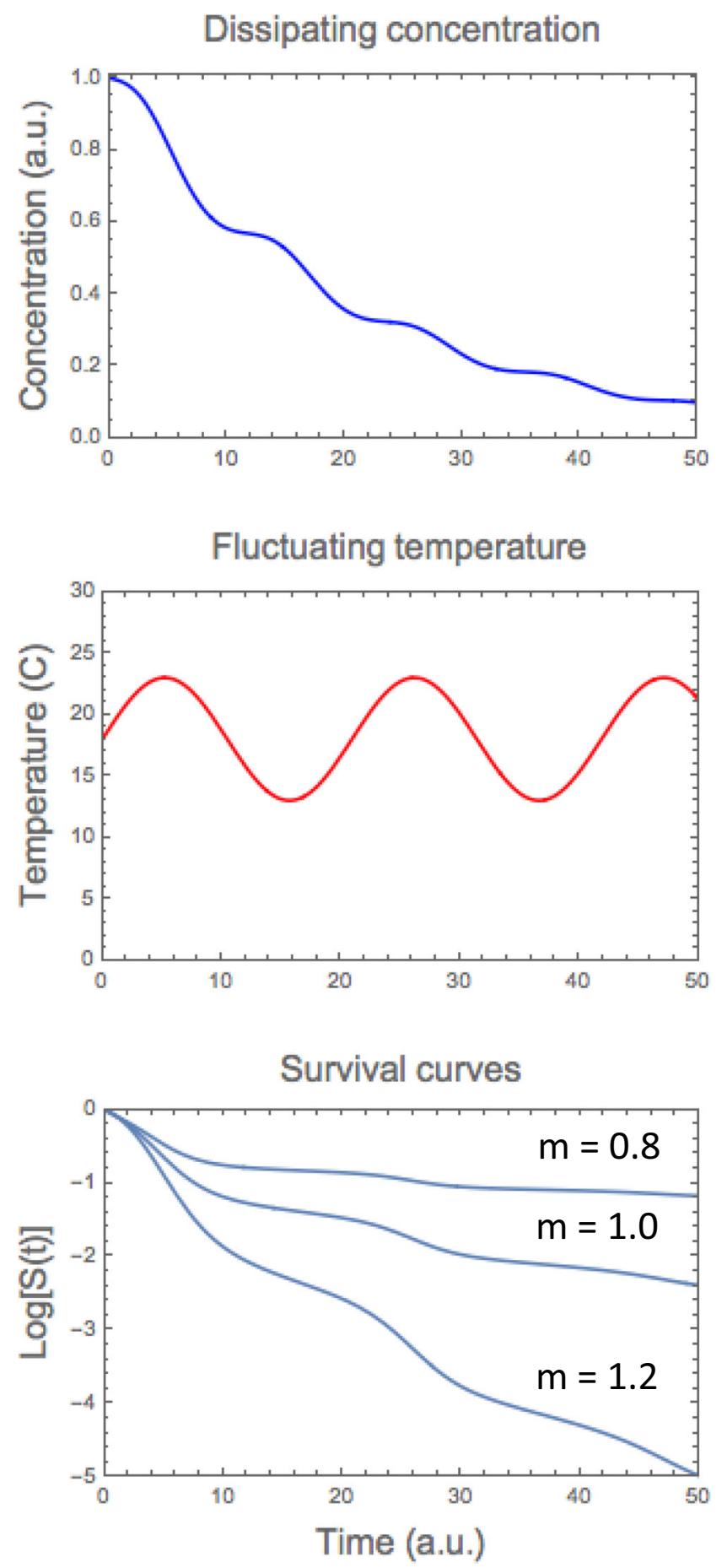

Fig. 8 Simulated dynamic survival curves (bottom) under simultaneously dissipating disinfectant agent (top) and fluctuating temperature (middle) generated with the expanded Weibullian dynamic model (Eq. 16) with three shape factors $(\mathrm{ms})$

the potentially dramatic effect that the shape factor $m$ might have if it is allowed to vary while the other model parameters remain fixed. The figure also demonstrates that the assumption of first-order inactivation kinetics, while the actual kinetics is not, might be fairly harmless initially may result in large discrepancies after a longer time. 


\section{The Fermi Distribution}

None of the already mentioned survival models can be considered inherently superior and two or more of them can be frequently used interchangeably. The same can be said about other unimodal distributions not mentioned. Statistical fit criteria alone, as already stated, are insufficient to establish a model's uniqueness. A notable exception to the above is the already mentioned Fermi distribution function and similarly constructed models. They too can be indistinguishable from the Weibull or lognormal distributions when describing a wide distribution as shown in Fig. 2 (right). The Fermi model's particular and clear advantage is its ability to describe very steep doseresponse or survival curves that resemble a step function, those of individual microbes included. [The function was proposed by Fermi to describe the mass density distribution within an atom, which at the nucleus edge drops almost vertically to almost zero.] When used as a dose-response or survival curve model it can be written in the form [33, 40].

$S(x)=\frac{1}{1+\operatorname{Exp}\left[\frac{x-x_{c}}{a}\right]}$

where $x$ is the dose (however defined) or time, $x_{\mathrm{c}}$ a marker of the curve's inflection point, where $S\left(x_{\mathrm{c}}\right)=1 / 2$, and $a$ the spread measure. If $a$ has any arbitrary value which is very small relative to $x-x_{\mathrm{c}}$, then it is easy to show that Eq. 17 produces a curve that for all practical purposes looks like a step drop. Yet, the Fermi distribution function is still a continuous function that has algebraic derivatives and can be integrated. As the value of $a$ increases, so does the spread around $x_{\mathrm{c}}$ (the variance) and eventually, the Fermi distribution becomes almost indistinguishable from the more familiar unimodal distribution. Notice that since

$\log [S(x)]=-\log \left[1+\operatorname{Exp}\left[\frac{x-x_{c}}{a}\right]\right]$

then when $x<<x_{\mathrm{c}}, \log [S(x)] \approx 0$, and when $x>>x_{\mathrm{c}}$, $\log [S(x)] \approx-\left(x-x_{\mathrm{c}}\right) / a$ as shown in Fig. 6 (right). But unlike the $0<x<\infty$ range of the Weibull or lognormal distribution, the Fermi distribution's range is $-\infty<x<\infty$. Consequently, $S(0)$ is always $<1$ and $\log [S(0)]<0$. However, this inevitable gap is usually negligibly small and has no practical implications, especially when dealing with highly scattered experimental data.

\section{The CT (or Ct) Concept}

According to Wikipedia: "CT Values are an important part of calculating disinfectant dosage for the chlorination of drinking water. A CT value is the product of the concentration of a disinfectant (e.g., free chlorine) and the contact time with the water being disinfected. It is typically expressed in units of
mg-min/L." The entry also has a table listing CT values of free chlorine (applied to Giardia cysts) in water to accomplish 1, 2, and 3 "log inactivation."

Despite its intuitive appeal and wide use in water disinfection (e.g., $[1,49])$, the CT concept is a peculiar one. The physical meaning of dosage expressed as having $M \theta / L^{3}$ (mass $\times$ time $\div$ volume) dimensions, which is equivalent to mass per volumetric flow rate, $M /\left(L^{3} / \theta\right)$, is at best unclear. Had all disinfection processes been static and followed first-order kinetics as described by the Chick-Watson model with $n=1$, then there would have been a unique universal relationship between the accomplished decimation, expressed in terms of the targeted microbe's log survival ratio, and CT. This is shown schematically in Fig. 9 (left). But for any (positive) $n \neq 1$, this will no more be the case and the accomplished level of inactivation will inherently depend on the disinfectant's concentration as shown schematically in Fig. 9 (right). In other words, since the ChickWatson model's equation can be written as $\log [S(t)]=-$ $k C^{n-1} C t$, then whenever $n \neq 1$, the slope of the $\log [S(t)]$ versus $C t$ relationship, i.e., $-k C^{n-1}$, is not constant but a function of the concentration $C$. The situation is further complicated if the static survival curve is not log-linear, as where it follows Hom's model. In that case, the model equation can be written as $\log [S(t)]=-k C^{n-1} t^{m-1} C t$ and therefore where $n \neq 1$ and $m \neq$ 1, the slope of the $\log [S(t)]$ versus $C t$ relationship, $-k C^{n-1} t^{m-1}$, will depend on both concentration and time. Similar claims can be made for models based on nonlinear kinetics such as following the Weibullian model.

Notice that the dosage is similarly defined for UV light disinfection, i.e., dose $=$ light intensity $\times$ time of exposure. But since UV light's intensity is expressed as power per unit area, the dose so defined has energy per area dimension and units, and hence is not an issue as in chemical disinfection.

\section{Stochastic (Probabilistic) Versus Deterministic Inactivation Models}

The survival kinetics models presented and discussed in the previous sections were originally devised for and tested with

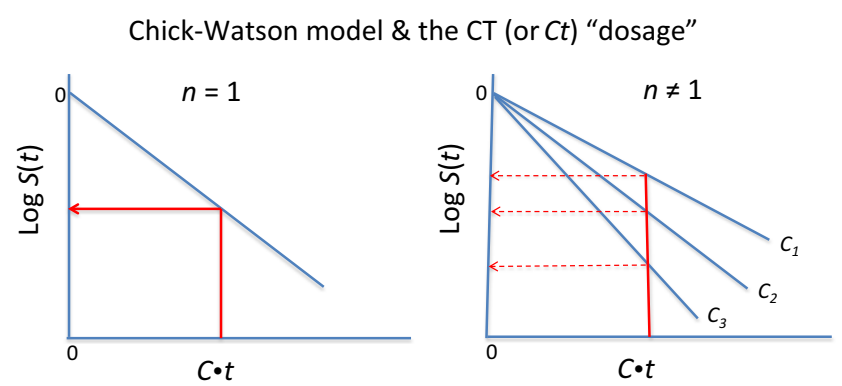

Fig. 9 The problem with CT (or $C t$ ) as a dosage measure: Only with the Chic-Watson model with $n=1$ is there a unique correspondence between the CT and the accomplished drop in the targeted microbe's survival ratio 
very large microbial populations reduced through disinfection by several orders of magnitude. They are all deterministic and expressed in the form of continuous functions. "Deterministic" in our context is manifested in that the survival curve is reproducible (despite that the individual counts themselves can be somewhat scattered, the main reason for their replication) and that when presented in the form of the survival ratio or its logarithm versus time relationship, the initial number is unimportant as long as there are enough microbes to be counted. [There are special cases where the initial inoculum size can be an issue but these should not concern us here.] But the same deterministic models that are applicable to large microbial populations may not be always applicable to very small microbial populations, especially when they are subjected to a marginal disinfection treatment. This is because at the individual microbe's level, even apparent continuity no more exists and probability plays a central role in whether it will survive or succumb after a given time.

Reports on the use of discrete stochastic (probabilistic) models of microbial inactivation are rather scarce in the literature on disinfection and food preservation. They will be briefly introduced here to alert the reader to their existence and potential explanatory power. (More detailed information can be found in $[11,12,24]$.)

Consider the simplest case where the targeted individual virus, bacterium, or other microbe can be in only two states, viable or inactivated, as shown schematically on the left side of Fig. 10. If it is viable at time 0 , then after time $\Delta t_{1}$ of exposure to the disinfectant, it can be either inactivated with a probability $P_{\mathrm{m}}(1)$ or remain viable with a probability $1-$ $P_{\mathrm{m}}(1)$. If it is still viable, then after another time interval $\Delta t_{2}$ (which can but need not be equal to $\Delta t_{1}$ ), it can be either inactivated with a probability $P_{\mathrm{m}}(2)$ or remain viable with a probability $1-P_{\mathrm{m}}(2)$, and so forth after $\Delta t_{3}, \Delta t_{4} \ldots \Delta t_{i} \ldots$ until it is finally inactivated and leaves the game. [Actually, the $P_{\mathrm{m}}(i) \mathrm{s}$ are probability rates, i.e., probability per unit time. To avoid this issue, we will assume unit time intervals, i.e., that each and every $\left.\Delta t_{i}=1.\right]$ In a group or population of $N_{0}$ microbes exposed to a lethal agent, each individual member plays the same "game." Therefore, if we count the total number of survivors after each $\Delta t_{i}$, i.e., $N(i)$, and plot this number versus time, we are actually constructing the group's survival curve. Examples of three hypothetical $P_{\mathrm{m}}(i)$ versus $i$ scenarios are shown in Fig. 10.

\section{Inactivation probability $\left(\boldsymbol{P}_{\mathrm{m}}\right): \quad$ Constant}

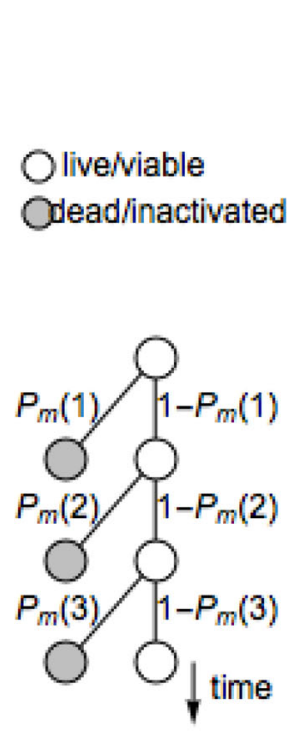

Sigmoidally dropping

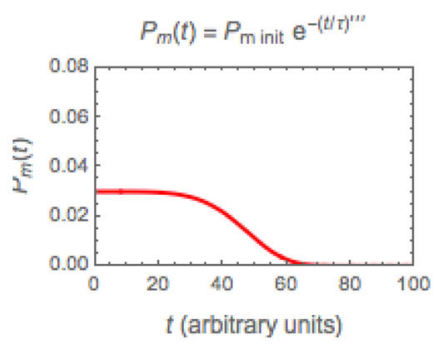

bitrary units)

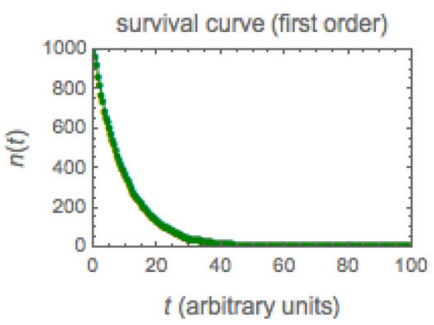

log survival (first order)
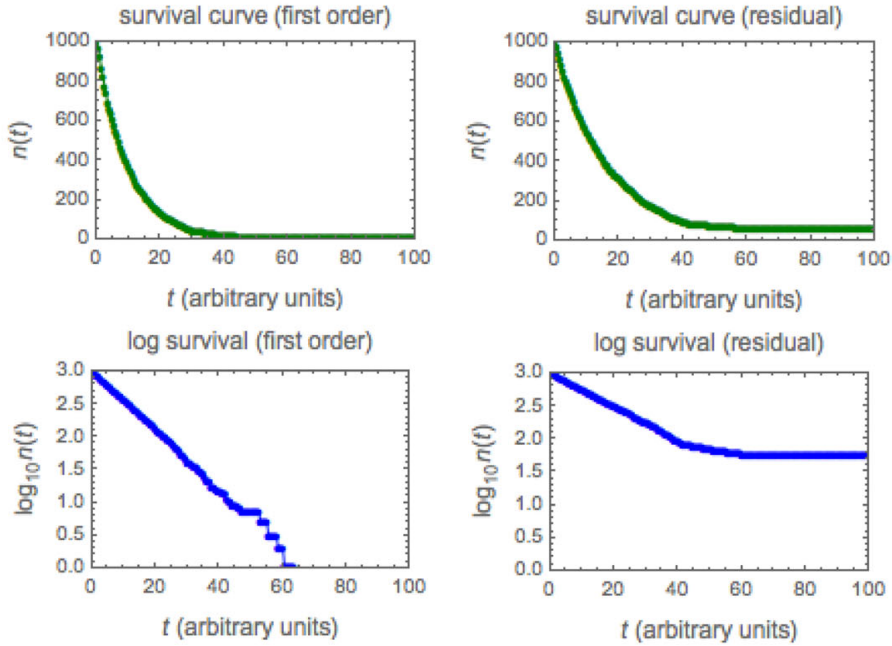
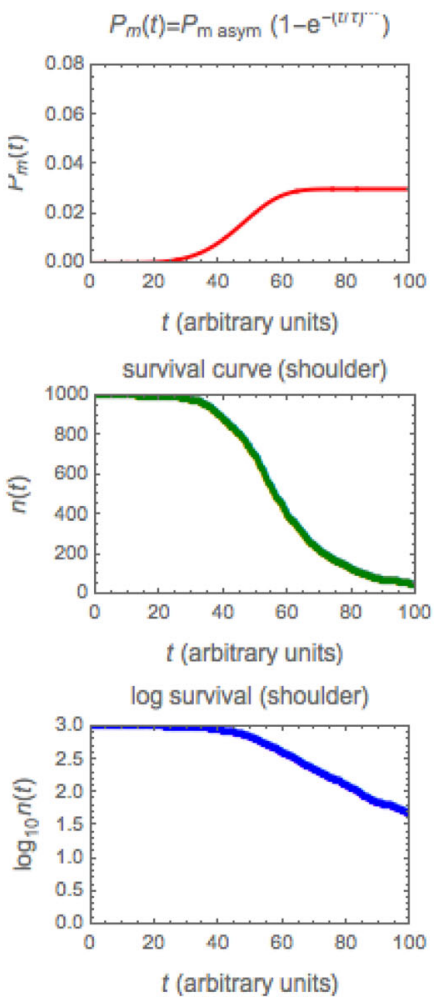

row: The corresponding survival curve plotted on linear coordinates. Bottom row: The corresponding survival curve plotted on log-linear coordinates. Notice that the left column, constant $P_{\mathrm{m}}(t)$, is equivalent to first-order kinetics

Fig. 10 Three examples of survival curves generated for 1000 individual microbes with the simplest stochastic model shown schematically on the left. $P_{\mathrm{m}}(i)$ is the inactivation/mortality probability per time unit. Top row: The inactivation/mortality probability history $\left(P_{\mathrm{m}}(t)\right.$ versus time $)$. Middle 
As has been shown [12,24], adjustment of the $P_{\mathrm{m}}(i)$ versus $i$ relationship can be used to produce almost all commonly encountered microbial inactivation patterns (see below). For the scenarios described in the figure, we assumed that all members of the group share the same set of probabilities $P_{\mathrm{m}}(i) \mathrm{s}$ and that these are functions of the exposure time only. Notice that because the process is purely probabilistic even with the same shared probability $P_{\mathrm{m}}(i)$ at each step, some individuals will survive longer than others before they perish too. Therefore, and consistent with observation, a survival curve so constructed need not be perfectly smooth even in principle, and need not be exactly reproducible (see below). However, it can be shown that as the number of individual microbes increases, their survival curve becomes progressively smoother and ever more deterministic $[12,24]$. What's also very important here is that the temporal changes in the $P_{\mathrm{m}}(i) \mathrm{s}$ can be extracted from conventional experimental survival curves obtained with large microbes populations and used to simulate the fates of small groups [24]. Such groups might be representative of realistic situations especially where a virulent pathogen's presence is less than massive.

Constant mortality probability, $P_{\mathrm{m}}(i)$ or $P_{\mathrm{m}}(t)$, throughout the disinfection, is equivalent first-order (log-linear) inactivation kinetics, as expected from its definition, except where we reach the last few survivors. At that time, see Fig. 10 (left column), the curve's continuation becomes jagged, and irreproducible, a clearer manifestation of the process's stochastic nature. The irreproducibility becomes dramatic when dealing with very small microbial populations or reaching a small number of survivors as demonstrated in Figs. 11 and 12 . Each figure shows the simulated fates of three identical groups of 20 individuals having exactly the same $P_{\mathrm{m}}(i) \mathrm{s}$; constant ("first-order kinetics") in Fig. 11 and decreasing (Weibullian where $m<1$ ). The three survival curves are shown in Fig. 12 are not only dissimilar but they can also be hardly recognized as being produced by the very same first-order inactivation kinetics. Also, and in contrast with and classic first-order kinetics theory, the stochastic model allows for the complete elimination of the targeted population in finite time. As demonstrated in Fig. 12, the same is true for Weibullian inactivation, including where it indicates tailing, i.e., where the semilogarithmic survival curve has upper concavity $(m<1)$ !

With different patterns of temporal changes in the $P_{\mathrm{m}}(i) \mathrm{s}$, as already mentioned, one can generate almost every known kind of inactivation kinetics. The plots in Figs. 10, 11, and 12 were produced with the freely downloadable Wolfram Demonstration (https://demonstrations.wolfram.com/ ProbabilisticModelForMicrobialMortality/), which allows the choice of four types of $P_{\mathrm{m}}(i) \mathrm{s}$ and the initial microbe's number to vary between 1 and 5000. Weibullian inactivation patterns with upward or downward concavities can be generated by selecting the linearly rising or falling $P_{\mathrm{m}}(i)$ option and adjusting the slope [12], or by adjusting the probability range of the proper sigmoid pattern.
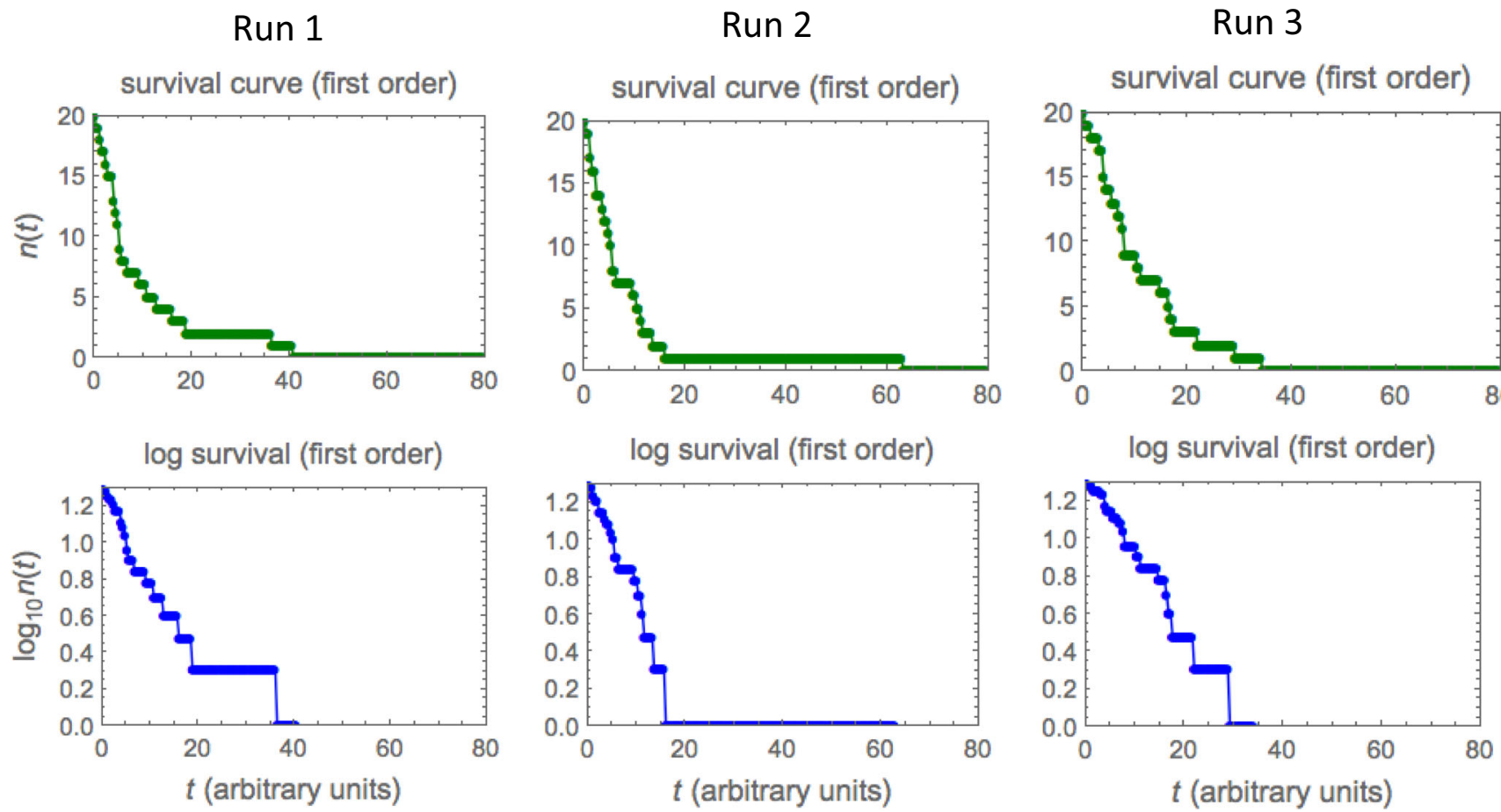

Fig. 11 Survival curves of three groups of 20 individual microbes (the three runs) each generated with the stochastic model shown schematically in Fig. 10 (left) having the same constant inactivation/mortality probability per time unit, $P_{\mathrm{m}}(t)=0.05$, plotted on linear (top row) and

log-linear (bottom row) coordinates. Notice the curve's irreproducibility, in which the expected first-order kinetic pattern is hardly recognizable and in which each group is totally eliminated in a finite time 
Run 1
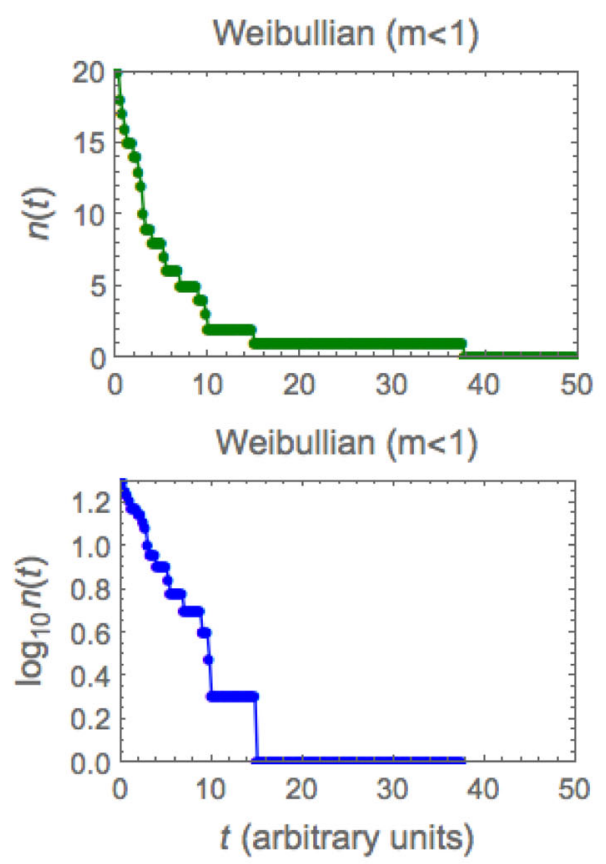

Fig. 12 Survival curves of three groups of 20 individual microbes each generated with the stochastic model shown schematically in Fig. 10 (left), whose $P_{\mathrm{m}}(t)$ continuously decreases (tailing) according to the Weibullian model where $m<1$, plotted on linear and log-linear coordinates, top and

A similar stochastic model with added division probabilities, $P_{\mathrm{d}}(i) \mathrm{s}$, introduced at each step can simulate resumed growth after insufficient treatment (of the same kind shown in Fig. 1) or the onset of inactivation after sustained growth [24]. At least in principle, stochastic models can also be written for scenarios that include cell division, injury and damage repair [36] and simultaneous activation and inactivation of bacterial spores [11].

It seems unlikely that stochastic model of disinfection will replace the conventional deterministic ones any time soon. But a probabilistic model even if only used for simulations can be a valuable tool to investigate the connection between the fates of individual virus units or bacterial cells and their observed manifestations at the population level.

\section{Concluding Remarks}

The notion of an underlying distribution function is well established in modeling type I dose-response curves but not so in modeling type II curves, where its relation to kinetics is yet to be universally acknowledged and accepted. Application of the popular Chick-Watson-Hom's disinfection model, especially in its published dynamic version, can be problematic on theoretical grounds. Its replacement by the static or dynamic version of the Weibullian, or a similar
Run 2

Run 3
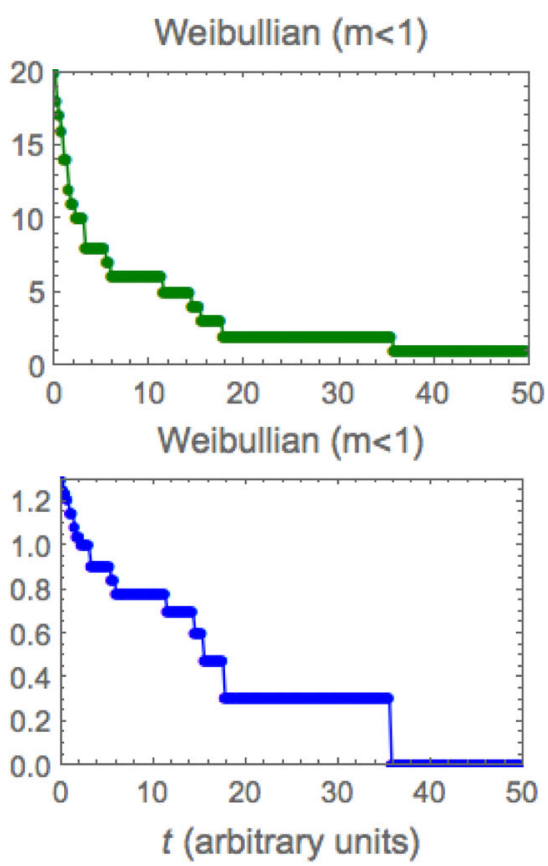

bottom, respectively. Notice that the curves' typical upward concavity can disappear when the survivors' numbers reach single digits and that they become totally irreproducible

kind of model, could resolve this predicament. Such a models' coefficients can be written in a manner that will account not only for the effect of a disinfectant's varying concentration or intensity consistently but will also for the effects of varying temperature and other ambient conditions. Experimental determination of such expanded models' coefficients may not be always feasible. But they can be used in simulations with assumed parameters to reveal patterns that would be otherwise difficult to predict.

The CT concept is only applicable to survival patterns that follow first-order kinetics where the rate constant is proportional to the disinfectant's concentration. It becomes problematic when the concentration scaling (power) is different than one $(n \neq 1)$. In the case of Hom's or other nonlinear inactivation patterns, the momentary survival ratio that corresponds to any $\mathrm{CT}$ value is concentration and time-dependent by definition. Therefore, its utility as a measure of a volatile chemical disinfectant's dosage should be reconsidered. Stochastic models of microbial inactivation, currently not widely employed, can be used to explain how different types of disinfection kinetics emerge. They also explain the irregularity and irreproducibility observed in the survival patterns of small groups of pathogens exposed to a disinfectant.

Although the emphasis in this review has been on viruses and bacteria, most if not all the discussion is also relevant to fungi, protozoa, and even worms. 
Acknowledgments The author thanks Mr. Mark D. Normand, for programming the Wolfram Demonstrations and other software on which much of the discussion is based, and to Dr. Matthew D. Moore for providing an important relevant piece of information.

\section{References}

1. Anon (2017) Enteric viruses in drinking water. Document for Public Consultation Health Canada (On the Internet)

2. Aragao GMF, Corradini MG, Normand MD, Peleg M (2007) Evaluation of the Weibull and log-normal distribution functions as survival models of Escherichia coli under isothermal and nonisothermal conditions. Int J Food Microbiol 19:243-257

3. Bosch A, Pinto RM, Guix S (2016) Foodborne viruses. Currnt Opinion Food Sci 8:110-119

4. Bozkurt H, D'Souza DH, Davidson PM (2015) Thermal inactivation kinetics of human norovirus surrogates and hepatitis A virus in Turkey deli meat. Appl Environ Microbiol 81:4850-5859

5. Brennecke M (2009) Disinfection kinetics of virus aggregates of bacteriophage MS2. Master Thesis. Laboratory of Environmental Chemistry, Ecole Polytechnique Federale de Lausanne. Lausanne, Switzerland

6. Brown WK, Wohletz KH (1995) Derivation of the Weibull distribution based on physical connection to the Rosin-Rammler and lognormal distributions. J Appl Phys 78:2758-2763

7. Chick H (1908) Investigation of the laws of disinfection. Epidemiol Infect

8. Buzrul S (2017) Evaluation of different dose-response models for high hydrostatic pressure inactivation of microorganisms. Foods 6(79):1-17

9. Chick H (1908) Investigation of the laws of disinfection. Epidemiol Infect 8:92-158

10. Cook N, Knight A, Richards GP (2016) Persistence and elimination of human norovirus in food and on food contact surfaces: a critical review. J Food Protect 79:1273-1294

11. Corradini MG, Normand MD, Eisenberg M, Peleg M (2010) Evaluation of a stochastic inactivation model for heat-activated spores of Bacillus spp. Appl Environ Microbiol 76:4402-4412

12. Corradini MG, Normand MD, Peleg M (2010) A stochastic and deterministic model of microbial heat inactivation. J Food Sci 75: R59-R70

13. Corradini MG, Peleg M (2003) A model of microbial survival curves in water treated with a volatile disinfectant. J Appl Microbiol 95:1268-1276

14. Ganguly P, Byrnea C, Breen A, Pillai SC (2018) Antimicrobial activity of photocatalysts: fundamentals, mechanisms, kinetics and recent advances. Appl Catalysis B: Environmental 225:51-75

15. Garba CP (2015) Disinfection. Chapter 29. In: Pepper IA, Gerba CP, Gentry TJ (eds) Environmental microbiology, 3rd edn. Elsevier, pp 645-662

16. Gil MM, Miller FA, Brandao TRS, Silva CLM (2017) Mathematical models for prediction of temperature effects on kinetic parameters of microorganisms' inactivation: tools for model comparison and adequacy in data fitting. Food Bioproc Technol 10:2208-2225

17. Guzel-Seydim ZB, Greene AK, Seydim AC (2004) Use of ozone in the food industry. LWT 37:453-460

18. Gyurek LL, Finch GR (1998) Modeling water treatment chemical disinfection kinetics. J Environ Eng 9:783-793

19. Haas CN, Joffe J, Anmangandla U, Tacangelo JG, Heath M (1996) Water quality and disinfection kinetics. J AWWA 88:95-103

20. Haas CN, Joffe J (1994) Disinfection under dynamic conditions: modification of Hom's model for decay. Environ Sci Technol 28: $1367-1469$
21. Haas CN, Kara SB (1984) Kinetics of microbial inactivation by chlorine - I Review of results in demand free systems. Water Res 11:1443-1449

22. Haas CN, Rose JB, Gerba C, Regli S (1993) Risk assessment of virus in drinking water. Risk Assess 13:545-552

23. Hijnen WAM, Beerendonk EF, Medema GJ (2006) Inactivation credit of UV radiation for viruses, bacteria and protozoan (oo)cysts in water: a review. Water Res 40:3-22

24. Horowitz J, Normand MD, Corradini MG, Peleg M (2010) A probabilistic model of growth, division and mortality of microbial cells. Appl Environ Microbiol 76:230-242

25. Jones RM, Su Y-M (2015) Dose-response models for selected respiratory infectious agents: Bordetella pertussis, group a Streptococcus, rhinovirus and respiratory syncytial virus. BMC Infect Diseases 15:90 (1-9)

26. Joshi K, Mahendran R, Alagusundaram K, Norton T, Tiwari BK (2013) Novel disinfectants for fresh produce. Trends Food Sci Technol 34:54-61

27. Kitajima M, Huamg Y, Watanabe T, Katayama H, Haas CN (2011) Dose-response time modelling for highly pathogenic avian influenza A (H5N1) virus infection. Letter Appl Microbiol 53:438-444

28. Lambert RJW, Johnston MD (2000) Disinfection kinetics: a new hypothesis and model for the tailing of log-survivor/time curves. J Appl Micobiol 88:907-913

29. Mattle MJ, Crouzy B, Brennecke M, Wigginton KR, Perona P, Kohn T (2011) Impact of virus aggregation on inactivation by peracetic acid an implications for other disinfectants. Environ Sci Technol 45:7710-7717

30. Messner MJ, Berger P, Nappier SP (2014) Fractional Poisson - a simple dose response model for human norovirus. Risk Anal 34: $1820-1829$

31. Misra NN, Tiwari BK, Raghavarao KSMS (2011) Nonthermal plasma inactivation of food-borne pathogens. Food Eng Rev 3: $159-170$

32. Najm I (2006) An alternative interpretation of disinfection kinetics. J AWWA 98:93-101

33. Peleg M (1996) Evaluation of the Fermi equation as a model of dose-response curves. Appl Microbiol Biotechnol 46:303-306

34. Peleg M (2000) Microbial survival curves - the reality of flat "shoulders" and thermal death times. Food Res Int 33:531-538

35. Peleg M (2006) Advanced quantitative microbiology for food and biosystems: models for predicting growth and inactivation. CRC Press, Boca Raton FL

36. Peleg M (2017) Modeling microbial inactivation by pulsed electric fields. In: Miklavcic D (ed) Handbook of electroporation. Springer, pp 1269-1286

37. Peleg M, Normand MD (2004) Calculating microbial survival parameters and predicting survival curves from non-isothermal inactivation data. Crit Rev Food Sci Nut 44:409-418

38. Peleg M, Normand MD, Corradini MG (2011) Construction of food and water borne pathogens' dose-response curves using the expanded Fermi solution. J Food Sci 76:R82-R89

39. Peleg M, Normand MD, Corradini MG (2012) The Arrhenius equation revisited. Crit Rev Food Sci Nut 52:830-851

40. Peleg M, Normand MD, Damrau E (1997) Mathematical interpretation of dose-response curves. Bull Math Biol 59:747-761

41. Peleg M, Normand MD, Horowitz J, Corradini MG (2007) An expanded Fermi solution for microbial risk assessment. Intrn J Food Microbiol 113:92-101

42. Peleg M, Normand MD, Horowitz J, Corradini MG (2011) Expanded Fermi solution for estimating the survival of ingested pathogenic and probiotic microbial cells and spores. Appl Environ Microbiol 77:312-319

43. Pressman JG, Wahman DG (2019) Understanding disinfection residuals. EPA Office of R\&D EPA.GOV 
44. Rossi S, Antonelli M, Mezzanotte V, Nurizzo C (2007) Peracetic acid disinfection: a feasible alternative to wastewater chlorination. Water Environ Res 79:341-350

45. Shereen MA, Khan S, Kazmi A, Bashir N, Siddique R (2020) COVID-19 infection: origin, transmission, and characteristics of human coronaviruses. J Adv Res 24:91-98

46. Sigstam T, Rohatschek A, Zhong Q, Brennecke M, Kohn T (2014) On the cause of the tailing phenomenon during virus disinfection by chlorine dioxide. Water Res 48:82-89

47. Sigstam T, Gannon G, Cascella M, Pecson BM, Wigginton KR, Kohn T (2013) Subtle differences in virus composition affect disinfection kinetics and mechanisms. Appl Envirn Microbiol 79: 3455-3467

48. Teunis PEM, Havelaar AH (2000) The beta Poisson dose-response model is not a single-hit model. Risk Anal 20:513-520

49. Thurston-Enriquez JA, Haas CN, Jacangelo J, Greba CP (2005) Inactivation of enteric adenovirus and feline calicivirus by chlorine dioxide. Appl Environ Microbiol 71:3100-3105

50. Van Boekel MAJS (2002) On the use of the Weibull model to describe thermal inactivation of microbial vegetative cells. Intnl $\mathbf{J}$ Food Microbiol 74:139-159

51. Van Boekel MAJS (2008) Kinetic modeling of food quality: a review. Comp Rev Food Sci Food Safety 7:144-158
52. Verhaelen K, Bouwknegt M, Lodder-Verschoor F, Rutjes SA, de Roda Husman AM (2012) Persistence of human norovirus GII.4 and GI.4, murine norovirus, and human adenovirus on soft berries as compared with PBS at commonly applied storage conditions. Intnl J Food Microbiol 160:137-144

53. Watanabe T, Bartrand TA, Omura T, Haas CN (2012) Doseresponse assessment for influenza A virus based on data sets of infection with its live attenuated reassortants. Risk Anal 32:555565

54. Watson HE (1908) A note on the variation of the rate of disinfection with change in the concentration of the disinfectant. Epidemiol Infect 8:536-542

55. Weir MH, Mitchell J, Flynn W, Pope JM (2017) Development of a microbial dose response visualization and modelling application for QMRA modelers and educators. Environ Modelling Software 88: 74-83

56. Xie G, Roiko A, Stratton H, Lemckert C, Dunn PK, Mengersen K (2017) Guidelines for use of the approximate beta-Poisson doseresponse model. Risk Anal 37:1388-1402

Publisher's Note Springer Nature remains neutral with regard to jurisdictional claims in published maps and institutional affiliations. 